\title{
Versatility of Aloe Vera in Dentistry- A review
}

\author{
**Dr.Tulsi Subramaniam, **Dr.Arun Subramaniam, ***Dr.Asha Chowdhery, \\ $* * * *$ Dr.Sreeja Das, $* * * * *$ Dr.Mishali Gill \\ *Professor, Dept. of Prosthodontics \& Crown \& Bridge, Dr.D.Y.Patil Dental College \& Hospital, \\ DPU, Pune, Maharashtra \\ **Professor \& Head, Dept. of Oral Medicine \& Radiology, Dr.D.Y.Patil Dental College \& Hospital, \\ DPU, Pune, Maharashtra \\ ***Professor, Dept. of Oral Medicine \& Radiology, Dr.D.Y.Patil Dental College \& Hospital, \\ DPU, Pune, Maharashtra \\ ****PG student, Dept. of Oral Medicine \& Radiology, Dr.D.Y.Patil Dental College \& Hospital, \\ DPU, Pune, Maharashtra \\ *****PG student, Dept. of Oral Medicine \& Radiology, Dr.D.Y.Patil Dental College \& Hospital, \\ DPU, Pune, Maharashtra
}

\begin{abstract}
The Aloe vera plant has been known and used for centuries for its medicinal and skin care properties. The name Aloe vera derives from the Arabic word "Alloeh" meaning shining bitter substance, while "vera" in Latin means true. The Greek scientists regarded Aloe vera as the universal panacea. Aloe vera has its uses in various systemic conditions like skin disorders (e.g. psoriasis), bowel disorders, diabetes and in hyperlipidemic patients. It has also been used in dentistry for its beneficial properties in various conditions like lichen planus, apthous stomatitis, oral submucous fibrosis, pulpotomy of primary teeth, prevention of dry sockets, obturation of primary teeth, disinfection of irrigation units, bleeding and painful gums, disinfection of gutta percha cones, burning mouth syndrome and in radiated head and neck cancer patients. However, future researches should be aimed to determine its best method of preparation, optimal concentration, time of application and effects on the oral cavity.
\end{abstract}

\section{Introduction}

Aloe vera is the oldest medicinal plant ever known and the most applied medicinal plant worldwide. ${ }^{1}$ Aloe (Aloe vera) is an important and traditional medicinal plant belonging to the family Liliaceae. There are over 400 species of Aloe Vera plants in the Lily Family. Aloe plants tend to grow $80-100 \mathrm{~cm}$ tall. $^{2}$ It is indigenous to Africa and Mediterranean countries. It is reported to grow wild in the islands of Cyprus, Malta, Sicily, Canary cape, Cape Verde and arid tracts of India. This is a hardy perennial tropical plant that can be cultivated in drought prone areas and is one of the crops whose potential is yet to be exploited, despite being identified as 'a new plant resource with the most promising prospects in the world.' (Fig 1) In India, it is scattered in the wild, along the coast of southern India.It is a powerful detoxifier, antiseptic and tonic for the nervous system. It also has immune-boosting and anti-viral properties. ${ }^{1}$ The cosmetic and medicinal products are made from the mucilaginous tissue in the centre of the plant called aloe vera gel. (Fig 2) The peripheral bundle of sheath cells produces intensely bitter, yellow latex, commonly termed as aloes. Unlike aloes, AV gel contains no anthraquinones, which are responsible for the strong laxative effects of aloes. The pharmacological actions of Aloe vera were studied in-vitro and in-vivo including anti-inflammatory, anti-arthritic, antibacterial and hypoglycemic effects. ${ }^{3}$ More than 75 active ingredients from inner gel (Aloe vera leaf pulp and exudates) have been identified including vitamins, minerals, enzymes, sugars, anthraquinones or phenolic compounds, lignin, saponins, sterols, amino acids and salicylic acid. ${ }^{4}$ 


\begin{tabular}{|c|c|}
\hline Class & Compounds \\
\hline Vitamins & B1, B2, B6, C, A ( $\beta \beta$-carotene), choline, folic acid, $\alpha \alpha+t o c o p h e r o l$ \\
\hline Enzymes & $\begin{array}{l}\text { Alkaline phosphatase, amylase, carboxypeptidase, catalase, bradikinase, cyclooxidase, peroxidase, } \\
\text { carboxy-peptidase, cyclooxygenase, lipase, oxidase, phosphoenolpyruvate carboxylase, superoxide } \\
\text { dismutase }\end{array}$ \\
\hline $\begin{array}{l}\text { Anthraquinones/ } \\
\text { anthrones }\end{array}$ & $\begin{array}{l}\text { Aloe-emodin, aloetic-acid, anthranol, aloin A and B (or collectively known as barbaloin), isobarbaloin, } \\
\text { emodin, ester of cinnamic acid }\end{array}$ \\
\hline Inorganic compounds & $\begin{array}{l}\text { Calcium, chlorine, chromium, copper, iton, magnesium, manganese, selenium, zinc, potassium, } \\
\text { phosphotous, sodium }\end{array}$ \\
\hline Carbohydrates & $\begin{array}{l}\text { Pure mannan, acetylated mannan, acetylated glucomannan (acemannan), galactan, } \\
\text { glucogalactomannan, galactogalacturan, galactoglucoarabinomannan, arabinogalactan,pectic } \\
\text { substance, xylan, cellulose }\end{array}$ \\
\hline Saccharides & Mannose, glucose, L-thamnose, aldopentose \\
\hline $\begin{array}{l}\text { Organic compounds } \\
\text { and lipids }\end{array}$ & $\begin{array}{l}\text { Arachidonic acid, } / \text {-linolenic acid, steroids (campestrol, cholesterol, } \beta \beta \text {-sitosterol), triglicerides. } \\
\text { triterpenoid, gibberillin, lignins, potassium sorbate, salicylic acid, uric acid }\end{array}$ \\
\hline Chromones & 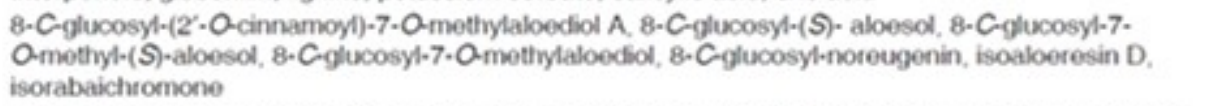 \\
\hline $\begin{array}{l}\text { Nonossential and } \\
\text { essential amino acids }\end{array}$ & $\begin{array}{l}\text { Alanine, arginine, aspartic acid, glutamic acid, glycine, histidine, hydroxyproline, isoleucine, leucine. } \\
\text { lysine, methionine, phenylalanine, proline, threonine, tyrosine, valine }\end{array}$ \\
\hline
\end{tabular}

Active ingredients of Aloe vera leaf pulp and exudates are depicted in the table above: ${ }^{4}$

\section{Actions of aloe vera gel:}

1) Healing properties: Countless studies have demonstrated the healing powers of aloe vera gel. A 1996 study reported that a high molecular weight polypeptide constituent from the gel demonstrated a healing effect on excisional wounds in rats. ${ }^{5}$ Glucomannan, a mannose-rich polysaccharide and gibberellin, a growth hormone, interacts with growth factor receptors on the fibroblast, thereby stimulating its activity and proliferation, which in turn significantly increases collagen synthesis after topical and oral Aloe vera application. ${ }^{6}$ In 1991, Thompson reported that topical application of the aloe vera-derived allatonin gel stimulated fibroblastic activity and collagen proliferation. ${ }^{7}$ Yagi et al. reported that Aloe vera gel contains a glycoprotein with cell proliferating-promoting activity, while Davis et al. noted that Aloe vera gel improved wound healing by increasing blood supply (angiogenesis), which increased oxygenation as a result. ${ }^{8}$

2) Protective effects: Aloe vera gel has been reported to have a protective effect against radiation damage to the skin. ${ }^{9}$ On skin exposed to UV and gamma radiation, following the administration of aloe vera gel, an antioxidant protein, metallothionein, is generated in the skin, which scavenges hydroxyl radicals and prevents suppression of superoxide dismutase and glutathione peroxidase in the skin. ${ }^{10}$ Glutathione peroxide activity, superoxide dismutase enzymes, and a phenolic antioxidant were found to be present in Aloe vera gel, which may be responsible for these antioxidant efects. Apart from these, it also contains A, C, and E vitamins. ${ }^{8}$

3) Anti-inflammatory action: Aloe vera inhibits the cyclooxygenase pathway and reduces prostaglandin E2 production from arachidonic acid. ${ }^{6}$ Recently, the novel anti-inflammatory compound called C-glucosyl chromone was isolated from gel extracts. ${ }^{10}$ In addition, the peptidase bradykinase was isolated from Aloe and shown to break down the bradykinin, an inlammatory substance that induces pain. ${ }^{8}$

4) Effects on the immune system: Alprogen inhibit calcium influx into mast cells, thereby inhibiting the antigen-antibody-mediated release of histamine and leukotriene from mast cells. ${ }^{9}$ Acemannan stimulates the synthesis and release of interleukin-1 (IL-1) and tumor necrosis factor from macrophages which in turn initiates an immune attack that result in necrosis and regression of the cancerous cells. ${ }^{6}$

5) Antitumor Effect: The two fractions from Aloe vera that are claimed to have anticancer efects include glycoproteins (lectins) and polysaccharides. Diferent studies indicated antitumor activity for Aloe vera gel in terms of reduced tumor burden, tumor shrinkage, tumor necrosis and prolonged survival rates. ${ }^{8}$ An induction of glutathione S-transferase and an inhibition of tumour promoting effect of phorbol myristic acetate has also been reported which suggests possible benefit of using aloe gel in cancer chemotherapy. ${ }^{7}$

6) Antibacterial Property: Streptoccocus pyogenes and Streptococcus faecalis are two microorganisms that have been inhibited by aloe vera gel. ${ }^{5}$ Aloe vera gel reportedly was bactericidal against Pseudomonas aeruginosa while acemannan prevented it from adhering to human lung epithelial cells in a monolayer culture. ${ }^{8}$

7) Antifungal Property: A processed aloe vera gel preparation reportedly inhibited the growth of Candida albicans. ${ }^{5}$ The purified Aloe protein has been found to exhibit potent antifungal activity against Candida paraprilosis, Candida krusei and Candida albicans. ${ }^{11}$

8) Antiviral Property: This action may be direct and indirect: indirect due to stimulation of immune system, and direct due to aloe emodin. Aloe vera is virucidal to Herpes simplex virus type 1 and type 2, Varicella zoster virus, pseudorabies virus and inluenza virus according to the research of Thomson. The results indicated that aloe emodin directly affected both DNA- and RNA-containing enveloped viruses but had no effect on naked 
(unenveloped) viruses. ${ }^{8}$ A 1990 study noted that fractions of aloe vera gel containing lectins directly inhibited the cytomegalovirus proliferation in cell culture, perhaps by interfering with protein synthesis. ${ }^{5}$

9) Moisturizing and anti-aging effect: It was proposed that the Aloe vera gel formulations with higher concentrations $(0.25 \% \mathrm{w} / \mathrm{w}$ and $0.5 \% \mathrm{w} / \mathrm{w})$ improved skin hydration possibly by means of a humectant mechanism. Humectant mechanism means that the Aloe gel works by attracting water from the dermis below and by helping to keep this water bound in the stratum corneum. ${ }^{8}$ Aloe stimulates fibroblast which produces the collagen and elastin fibers making the skin more elastic and less wrinkled. ${ }^{10}$ It also has cohesive effects on the superficial flaking epidermal cells by sticking them together, which softens the skin. ${ }^{9}$

\section{Dental Applications}

Dental uses of Aloe vera are multiple. ${ }^{12}$ There are eight main uses of aloe vera in dental practice: ${ }^{7,9}$

1. Directly at the sites of periodontal surgery.

2. Applications to the gum tissues when they have been traumatized or scratched by toothbrush-dentifrice abrasion, sharp foods, dental floss, and toothpick injuries.

3. Chemical burns from accidents with aspirin.

4. Application at extraction sockets

5. Acute oral lesions such as herpetic lesions, aphthous ulcers, canker sores \& cracks occurring at the corners of our lips.

6. Chronic oral lesions such as Lichen Planus and Pemphigus

7. Patients with sore gums and teeth with dentures maladaptive may also benefit.

8. Can also be used around dental implants to control inflammation caused by bacterial contamination.

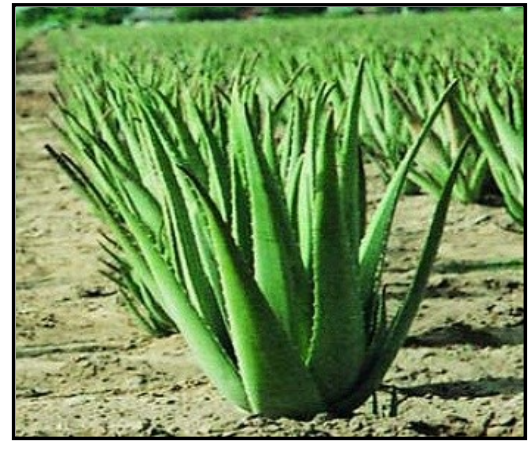

Fig 1: Aloe vera plant

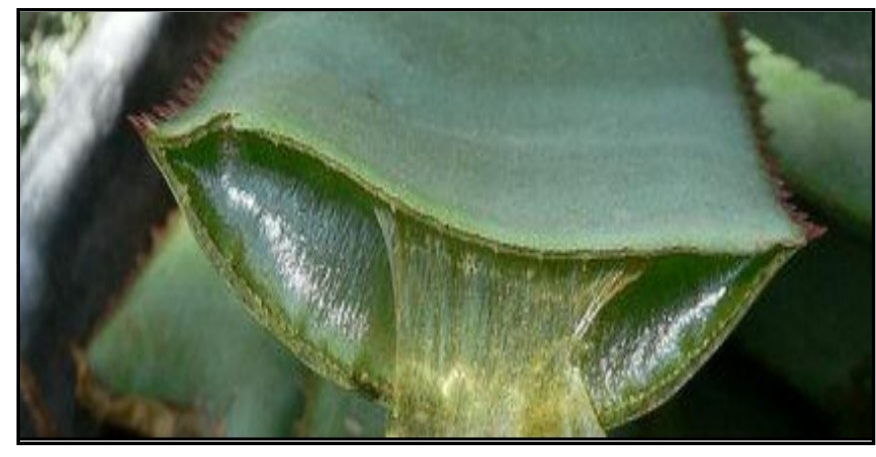

Fig 2: Inner gel of aloe vera plant

\section{Anticariogenic Activity:}

Mohammad Mehdi Fani found that, Aloe vera gel exerted strong bactericidal activity against both cariogenic and periodontopathic bacteria. Undiluted Aloe vera gel produced significant growth inhibition zones against all of the oral bacteria tested. In an experiment done, the mean MIC values for Aloe vera gel measured by the micro dilution method against clinical isolates of S.mutans, was $12.5 \mu \mathrm{g} / \mathrm{ml} .^{13}$

\section{Aloe vera in periodontal disease}

Aloe vera when used at full strength reduced accumulated plaque significantly. ${ }^{13}$ It is extremely helpful in the treatment of gingivitis and periodontitis. ${ }^{12}$ Aloe Vera greatly reduces the instances of gingival bleeding due to its soothing \& healing properties, reduces swelling and soft tissue edema. Hence it helps to restore gums to health. ${ }^{9}$ Aloe vera mouthwash can be an effective antiplaque agent and with appropriate refinements in taste and shelf life can be an affordable herbal substitute for chlorhexidine. ${ }^{8}$ Chlorhexidine, sodium hypochlorite, cetylpyridinium chloride and amine fluoride are widely used as mouthwashes ; immediate hypersensitivity reactions, toxicity, tooth staining and other side effects have been reported due to use at various times. Moreover, it has been reported that chlorhexidine and sodium hypochlorite are cytotoxic to human periodontal ligament cells, inhibit protein synthesis, and affect mitochondrial activity, thus, having detrimental effects on vital tissues. ${ }^{14}$ Subgingival administration of aloe vera gel results in improvement of periodontal condition. Aloe vera tooth gel tends to be less harsh on teeth, as it does not contain the abrasive elements typically found in commercial toothpaste; it is a great alternative for people with sensitive teeth or gums. Application directly to the sites of periodontal surgery along with periodontal dressing or to gum tissues when they have been traumatized by toothbrush-dentifrice abrasion, sharp foods, dental floss and toothpick injuries. ${ }^{10}$ 


\section{Apthous Stomatitis:}

Aloe vera oral gel is not only effective in decreasing the recurrent apthous stomatitis patient's pain score and wound size but also decreases the apthous wound healing period. ${ }^{11}$ Acute mouth lesions are improved by direct application in gel form on herpetic viral lesions or aphthous ulcers. It has been reported that acemannan hydrogel accelerates the healing of aphthous ulcers and reduces the pain associated with them. ${ }^{6}$ Acemannan, which is one of the polysaccharide components in Aloe vera, has been used for the treatment of oral apthous ulceration in patients who wish to avoid the use of steroid medication. US Food and Drug Administration has also found a derivative of Aloe vera an effective treatment alternative in treating oral ulcers ${ }^{11}$

\section{Oral lichen planus:}

Aloe Vera has also been used to treat lichen planus. The topical application of AV, three times a day improves the pain, the severity of the oral lesions, and the oral quality of life of the patients with OLP. ${ }^{15}$ As indicated by other studies, aloe vera can be used in dosages of two ounces aloe vera juice three times a day for three months. ${ }^{6}$ Steroids have remained the mainstay treatment modality in case of lichen planus, however, long term steroids therapy is associated with multiple systemic complications which provides Aloe vera an added advantage due its lesser side effects. Also, when compared with triamcinoline better results were obtained with topical Aloe vera. ${ }^{11}$

\section{Alveolar Osteitis:}

Currently, special medical bandages (SaliCept Patch) are available for intraoral use following extraction of teeth. ${ }^{8}$ SaliCept Patch is a freeze-dried pledget that contains Acemannan Hydrogel obtained from the clear inner gel of Aloe vera. After extraction, gauze saturated with Aloe vera when placed in socket and asked by the patient to bite on it, has shown improved healing \& formation of blood clot. ${ }^{9}$

\section{Denture Adhesive:}

Acemannan, a complex mannose carbohydrate, which is one of the main ingredients of the aloe vera gel was found to have good adhesive properties. ${ }^{11}$ It is this property that led to the production of prototype acemannan denture adhesives. These new denture adhesive formulations were evaluated for $\mathrm{pH}$ changes, cytotoxicity to human gingival fibroblasts and adhesive strength in both dry and wet conditions. In an experiment carried out, it was concluded that acemannan denture adhesive formulation with an initial $\mathrm{pH}$ value of 6.0 was an effective herbal substitute for traditional denture adhesives. ${ }^{8}$

\section{Aloe Vera In Endodontics:}

It has been used in root canal treatment as a sedative dressing and file lubrication during biomechanical preparation. $^{12}$ Aloe vera has proved to be a good obturative material for primary teeth. Retention of microorganisms within the dentinal tubules is thought to be a source of persistent endodontic infection. In persistent cases of root canal infection and in failed root canal treatments main organism identified is E. Faecalis. Recently herbal extracts are gaining attention in dentistry and Aloe vera has proved a good antibacterial (because of anthraquinones) against E. Faecalis if used in root canals. Aloe vera gel has been found to be effective in decontaminating GP cones within one minute. ${ }^{11}$

\section{Dental Implants:}

Aloe vera gel placed around dental implants is found effective to reduce inflammation. Aloe vera reduces inflammation by its antimicrobial \& anti-inflammatory effects. ${ }^{9}$

\section{Aloe Vera As A Tooth Gel:}

The majority of the antimicrobial effects of commercially available toothpastes can be attributed to their fluoride content, in the form of sodium monoflourophosphate (a concentration of 500-1,000 ppm). The aloe vera tooth gel has no added fluoride content but still exerts almost an equal amount of antimicrobial activity. ${ }^{16}$ Less harsh on teeth as it does not have the abrasive elements and hence is a better alternative for people with sensitive teeth or gums. ${ }^{9}$ Studies using aloe vera in toothpastes have shown that aloe vera tooth gel and the toothpastes were equally effective against Candida albicans, Streptococcus mutans, Lactobacillus acidophilus, Enterococcus faecalis, Prevotella intermedia and Peptostreptococcus anaerobius. Aloe Vera tooth gel demonstrated enhanced antibacterial effect against S. Mitis. ${ }^{10}$

\section{Aloe Vera As A Mouthwash:}

Mouthwash prevents radiation-induced mucositis by its wound healing and anti-inflammatory mechanism. ${ }^{9}$ Aloe vera mouthwash may not only prevent radiation-induced mucositis by its wound healing and anti-inflammatory mechanism, but also may reduce oral candidiasis of patients undergoing head and neck 
radiotherapy due to its antifungal and immunomodulatory properties. ${ }^{11}$ It is recommended that $1-3$ tablespoon of aloe vera juice be used as a mouthwash, then swallowed, three time daily. ${ }^{9}$

\section{Contraindications:}

In some cases contact dermatitis and hypersensitivity reactions after topical applications of Aloe vera gel have been noted. ${ }^{11}$ Hence it is contraindicated in cases of known allergy to plants in the Liliaceae family. ${ }^{9}$ Aloe should not be used during pregnancy or lactation except under medical supervision. Oral use of Aloe vera in children under 10 years of age is contraindicated. In diabetic patients, increased hypoglycemia might be seen in conjunction with oral antidiabetics or insulin. Aloe vera gel for systemic application is not recommended in combination with antidiabetic, diuretic, or laxative drugs; sevoflurane; or digoxin. ${ }^{11}$ Application of aloe to skin may increase the absorption of steroid creams such as hydrocortisone. It reduces the effectiveness and may increases the adverse effects of digoxin and digitoxin, due to its potassium lowering effect. Combined use of Aloe vera and furosemide may increase the risk of potassium depletion. It decreases the blood sugar levels and, thus, may interact with oral hypoglycaemic drugs and insulin. ${ }^{9}$

\section{SIDE EFFECTS:}

Topical: It may cause redness, burning, stinging sensation and rarely generalized dermatitis in sensitive individuals. It is preferred to apply it to a small area first to test for possible allergic reaction. ${ }^{9}$

Oral: Abdominal cramps, diarrhoea, red urine, hepatitis, dependency or worsening of constipation. Prolonged use has been reported to increase the risk of colorectal cancer. Laxative effect may cause electrolyte imbalances (low potassium levels). ${ }^{9}$

\section{Conclusion}

Aloe vera may find a promising role in various branches of dentistry in future. Proper diagnosis, knowledge of the traditional medicine and implementation of that knowledge to the treatment plan are important in ensuring success with this dental therapeutic agent. Standardization and quality assurance of aloe vera products plays a key area which needs to be focused. Further, controlled studies are also required in future to prove the effectiveness of aloe vera under various conditions. Also, the potential long term side effects of Aloe vera need to be studied and evaluated.

\section{References}

[1]. Rajeswari R et al, Aloe vera: The Miracle Plant Its Medicinal and Traditional Uses in India, Journal of Pharmacognosy and Phytochemistry.2012;1(4) :118-24

[2]. Aloe Vera: History, Science, and Medicinal Uses, www.HealingAloe.com:1-12

[3]. Kareman E S et al, A Self-controlled Single Blinded Clinical Trial to Evaluate Oral Lichen Planus after Topical Treatment with Aloe Vera, Journal of GHR, 2013; 2(4): 503-7

[4]. Gupta K V, Malhotra S, Pharmacological attribute of Aloe vera: Revalidation through experimental and clinical studies, Ayu, 2012; 33(2) :193-6

[5]. Richard LW, Aloe vera gel: Update for dentistry, Pharmacology Today. 2005: 6-9

[6]. Tanwar R et al, Aloe Vera and its uses in Dentistry, Indian J Dent Adv 2011;3(4): 656-8

[7]. Sundarkar P et al, Use of Aloe Vera in dentistry, Journal of Indian Academy of Oral Medicine and Radiology.2011; 23(3): S389-91

[8]. Sajjad A, Aloe vera: An Ancient Herb for Modern Dentistry-A Literature Review, Journal of Dental Surgery, 2014: 1-6

[9]. Meena M et al, Aloe vera - An Update for Dentistry, Journal of dentofacial sciences. 2013; 2(4): 1-4

[10]. Sambhav J, Rai R, Aloe-Vera: A Boon In Management Of Dental Disease, Int. J. Pharm. Res. Sci., 2014; 2(1): 18-24

[11]. Tayal E et al, Current Perspectives on Use of Aloe vera in Dentistry, European Journal of Medicinal Plants, 2014; 4(12): 1408-19

[12]. Singla S, Aloe Vera: Use of Herbal Plant in Dentistry: 1-2

[13]. Rathi S, Role of aloe vera in dental practice- a review, The Pharma Research, 2013; 10(1): 1-5

[14]. Karim B et al, Effect of Aloe vera Mouthwash on Periodontal Health: Triple Blind Randomized Control Trial, OHDM, 2014; 13(1): 14-19

[15]. Salazar-Sa 'nchez et al, Efficacy of topical Aloe vera in patients with oral lichen planus: a randomized double-blind study, J Oral Pathol Med, 2010; 39: 735-40

[16]. George D et al, Comparative evaluation of the antimicrobial efficacy of aloe vera tooth gel and two popular commercial toothpastes: An in vitro study, General Dentistry, 2009: 238-41 